\title{
X-ray-line coincidence photopumping in a potassium-chlorine mixed plasma
}

\author{
L. M. R. Hobbs $\odot,{ }^{*}$ D. Burridge, M. P. Hill, D. J. Hoarty, C. R. D. Brown, R. Charles $\odot$, \\ G. Cooper, S. F. James, L. A. Wilson, and W. Babbage \\ AWE Plc, Aldermaston, Reading, RG4 7PR, United Kingdom \\ P. W. Hatfield \\ Department of Physics, Clarendon Laboratory, University of Oxford, Parks Road, Oxford OX1 3PU, United Kingdom \\ P. Beiersdorfer $\odot$, J. Nilsen $\odot$, and H. Scott \\ Lawrence Livermore National Laboratory, Livermore, California 94550, USA \\ S. J. Rose $\odot^{\dagger}$ \\ Plasma Physics Group, The Blackett Laboratory, Imperial College London, Prince Consort Road, London SW7 2AZ, United Kingdom
}

(Received 18 December 2019; accepted 28 April 2020; published 21 May 2020)

\begin{abstract}
Exploiting the multiple long pulse capability and suite of x-ray diagnostics of the Orion laser, we have set out to explore line coincidence photopuming - the enhancement in population of an atomic level brought on by resonant absorption of $\mathrm{x}$ rays from a different emitting ion. Unlike previous work, the two ions are in the same plasma and so the experiment is an x-ray analog of the well-known Bowen resonance fluorescence mechanism that operates in astrophysical situations in the optical region. Our measurements have shown enhanced fluorescence in a chlorine plasma, attributable to line coincident photopumping from co-mixed potassium ions. To detect this relatively low signal-to-noise phenomenon, the data from multiple shots are combined, and the statistical method of bootstrapping is used to assign a confidence value to the measured enhancement, resulting in an estimate of the enhancement of $39 \pm_{18}^{16} \%$ compared to the null case, where no pumping occurs. The experimental results have been compared to coupled radiation-transport and radiation hydrodynamics simulations using the CRETIN code together with the NYM radiation hydrodynamics model and agreement has been found, with the simulations also predicting modest enhancement.
\end{abstract}

DOI: 10.1103/PhysRevA.101.053431

\section{INTRODUCTION}

Line coincidence photopumping (LCP) relies on radiation from an emitting ion being absorbed by a separate ion and promoting an electron to a higher state. This will occur when the emission lines are resonant, i.e., close enough in energy. LCP was one of the original mechanisms proposed for demonstrating lasing at $\mathrm{x}$-ray wavelengths $[1,2]$. Strong $\mathrm{Ly}_{\alpha}$ or $\mathrm{He}_{\alpha}$ emission from a laser produced plasma was sought to efficiently force a population inversion in a lasing ion, which then decays via intermediary states to the ground. It is the characteristic signal of this decay that we looked to measure in this experiment, where substitution of the pumping ion with an alternate dopant produces a null result for comparison.

LCP was originally suggested by Bowen as an explanation for enhanced fluorescence in astrophysical plasmas [3] and recently has had renewed interest as an explanation for enhanced x-ray emission from astrophysical sources, with the first astrophysical observations of resonant $\mathrm{x}$-ray photopump-

\footnotetext{
*lauren.hobbs@awe.co.uk.

†Also at Department of Physics, Clarendon Laboratory, University of Oxford, Parks Road, Oxford OX1 3PU, United Kingdom.
}

ing reported by Keenan et al., having been detected in solar flares [4].

Achieving lasing at $\mathrm{x}$-ray wavelengths in a plasma amplifier using LCP still remains elusive, with the shortest wavelength at which gain has been observed being a Be-like $\mathrm{C}$ line at $2163 \AA$ [5]. However, there has been some success in the demonstration of enhanced fluorescence attributable to line coincidence photopumping, where $\mathrm{x}$ rays produced by line radiation have been used to directly pump a resonant transition in physically separate laser produced plasmas [6-10]. In particular, Back et al. conducted experiments and radiation transfer calculations utilizing an aluminum $\mathrm{He}_{\alpha}$ pump, noting the advantages of this line in creating a large population over a wider temperature range than is possible for $\mathrm{Ly}_{\alpha}$ emission $[7,8]$. The only published example of population inversion is from Porter et al. [11], where a $Z$ pinch was employed as the driver in a demonstration of resonant photopumping based on a sodium-neon scheme.

\section{PUMPING SCHEME AND EXPERIMENTAL SETUP}

This work builds upon previously published results with a scheme proposed by Nilsen [1,12-16] and further developed by Rose [17,18], Al-Miev [19], and Beer [20] in which both 


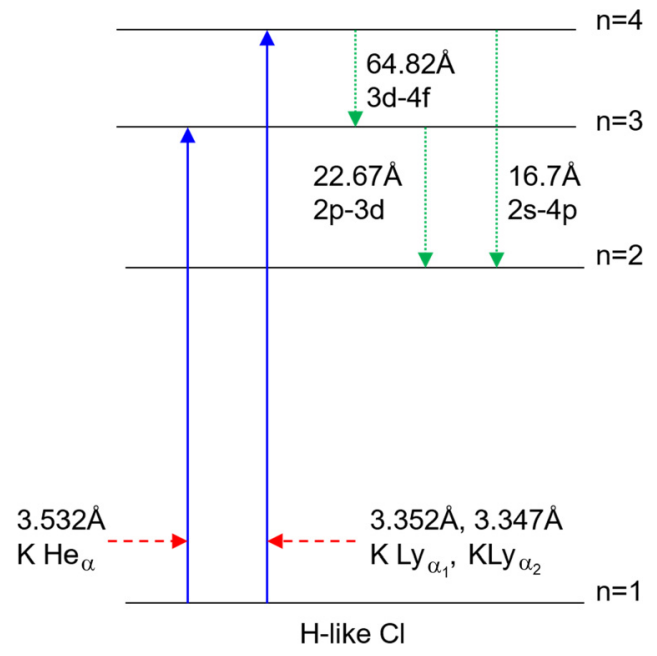

FIG. 1. Energy levels of $\mathrm{H}$-like $\mathrm{Cl}$ demonstrating the two line coincidences between $\mathrm{K} \mathrm{Ly}_{\alpha}$ and the 1-4 levels in $\mathrm{H}$-like $\mathrm{Cl}$ and $\mathrm{K} \mathrm{He}_{\alpha}$ and the 1-3 transition. Pumping lines are shown in red (dashed), pumped lines in blue (solid), and signature emission in green (dotted).

$\mathrm{Ly}_{\alpha}$ and $\mathrm{He}_{\alpha}$ radiation from $\mathrm{H}$-like and He-like potassium act as a resonant pump on two transitions in $\mathrm{H}$-like chlorine.

Figure 1 shows the energy level diagram for the coincident pairs. The incoming photon is absorbed, promoting an electron to a higher state. This then decays via intermediary states to the ground and it is the characteristic signal of this decay that provides a signature of enhancement. Substitution of the pumping ion with an alternate dopant (in our case sodium) produces a null result for comparison. Early simulations predicted modest gain, where concerns were raised that one coincidence is poisoned by the other, as the $\mathrm{He}_{\alpha}$ pump drives more elections into the $n=3$ state, reducing the potential population inversion required for significant gain for the $3 d-4 f$ transition. Instead, with the added advantages stated by Back et al. [7] we focus on the $\mathrm{He}_{\alpha}$ pump line and $\mathrm{K} \mathrm{He}_{\alpha}-\mathrm{Cl} \mathrm{Ly}{ }_{\beta}$ coincidence, with resultant signature emission from the $3 d_{5 / 2} \rightarrow 2 p_{3 / 2}$ transition at $22.67 \AA(546.9 \mathrm{eV})$. The tabulated energies of the pumping and pumped lines are 3.51 and $3.508 \mathrm{keV}$, respectively [21]. The $3 d_{5 / 2} \rightarrow 2 p_{3 / 2}$ transition represents the strongest line in this series. Fine structure of the $n=3$ and $n=2$ levels will merge into this transition due to line broadening and finite instrument resolution (approximately $0.33 \mathrm{eV}$ for this work); however, any difference between the signal and null case remains attributable to the pumping mechanism.

Steady-state modeling was conducted over a range of plasma densities and electron temperatures with the CRETIN nonlocal thermodynamic equilibrium atomic kinetics and radiation transfer code [22] using Flexible Atomic Code atomic data for potassium, chlorine, and sodium [23]. Calculations were conducted for the $\mathrm{KCl}$ plasma, with coupling between the pumping and pumped lines. Comparisons were then made to an $\mathrm{NaCl}$ plasma, this being the null case for the experimental work. One-dimensional cylindrical geometry was used, with spectral calculations made for a plasma of finite length.

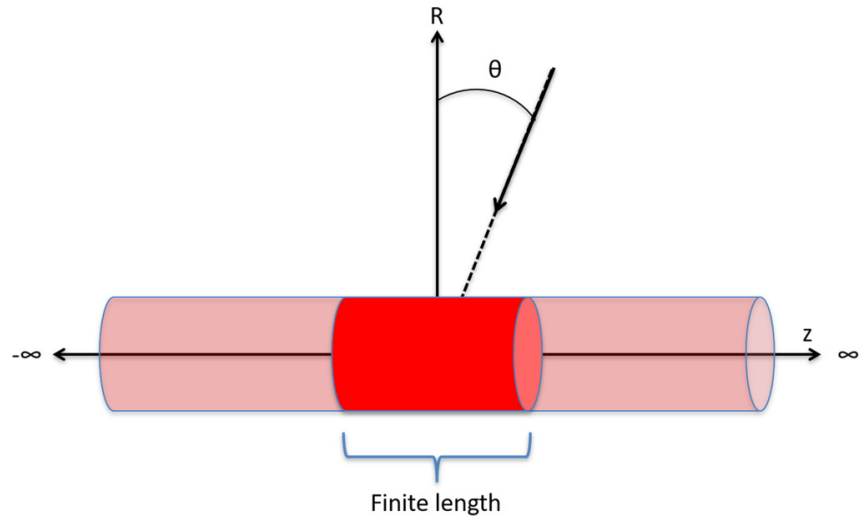

FIG. 2. Geometry employed for CRETIN simulation. CRETIN allows for the target dimensions and viewing angle to be specified, where the length of plasma in the line of sight will impact on the detected enhancement due to LCP. The plasma is simulated in cylindrical geometry with the finite length chosen to maintain areal density for the spectral calculations, as we scan across density in parameter space. The emission is "viewed" in the " $R$ " direction.

The geometry used is shown in Fig. 2. The length was varied so that the axial areal density was maintained.

Line transport modeling was conducted for the pumping and pumped line, significantly using the line-center energy separations taken from the National Institute of Standards and Technology energy level database [21]. The atomic physics data that CRETIN uses can be any level of fidelity. The data used here included splitting of the $2 p, 3 p$, and $3 d$ states with line-center transition energies of $550.32 \mathrm{eV}$ and $546.87 \mathrm{eV}$ for the $3 d_{3 / 2} \rightarrow 2 p_{1 / 2}$ and $3 d_{5 / 2} \rightarrow 2 p_{3 / 2}$ transitions, respectively.

For analysis, line intensities were integrated between the full width at half maximum of the $\mathrm{H}$-like $\mathrm{Cl} 2 p-3 d$ line, allowing comparison of the expected enhancement with the experimental data. Results are shown in Fig. 3, where optimum conditions predict an enhancement of $60 \%$. In reality, gradients and inhomogeneities in the plasma would prevent this from ever being achieved. Note that the pumping and pumped lines are not perfectly coincident, and treatment of line broadening and calculated intensities can greatly vary the predicted enhancement.

One-dimensional radiation hydrodynamic simulations conducted using the Lagrangian hydrocode NYM [24] have shown that in order to reach the required states of ionization, and to avoid collisional effects dominating over photopumping, electron temperatures of approximately $1 \mathrm{keV}$ and electron densities that correspond to $30 \mathrm{mg} \mathrm{cm}^{-3}$ are required for both ion species. For these conditions, we expect ion temperatures of approximately $500 \mathrm{eV}$. This value was taken for the CRETIN calculations, which ran assuming a constant ion temperature. The line shape, and therefore degree of coincidence, will change with ion temperature. The simulations were rerun using the high bound of ion temperature taken from NYM simulation. The resulting change in enhancement was within $10 \%$ of the value calculated at optimum pumping conditions of $500 \mathrm{eV}$ ion temperature.

For the schemes in which enhancement has been detected thus far, the pumping and pumped species exist in physically 


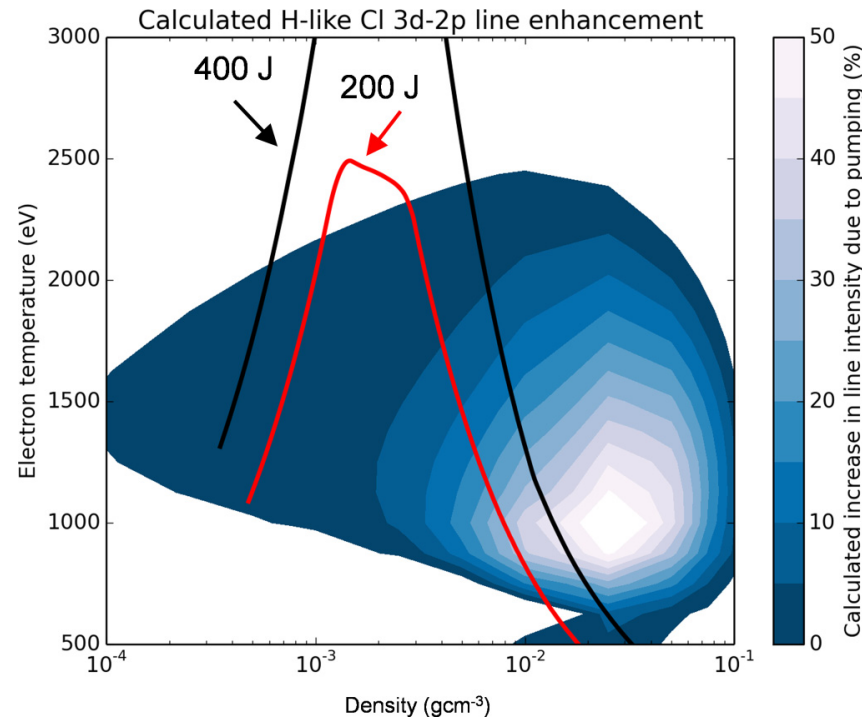

FIG. 3. Contour plot showing CRETIN calculated increases in line intensity of the $\mathrm{H}$-like $\mathrm{Cl} 2 p-3 d$ transition due to LCP in $\mathrm{KCl}$, compared to $\mathrm{NaCl}$. Also shown are the locus of points for average electron temperature and density of the $\mathrm{KCl}$ layer taken from 1D simulation using the NYM radiation hydrodynamic code. These represent the plasma conditions we expect to access for $400 \mathrm{~J}$ (black) and $200 \mathrm{~J}$ (red) laser drive energy incident on both sides of the buried layer target.

separate plasmas. The advantage of the scheme considered in this work over the others hitherto reported upon [6-11] is the possibility of creating a mixed plasma at the correct temperature and density for both $\mathrm{K}$ and $\mathrm{Cl}$. This allows us to neglect the differential Doppler shift that could otherwise take the lines out of coincidence, removing a large source of uncertainty from the calculations. It also avoids an additional experimental complexity that must be overcome, in scenarios where two very different plasma conditions would need to be achieved. This scenario of a mixed plasma is also more directly comparable to LCP in astrophysical sources, e.g., the $\mathrm{Ne}-\mathrm{Na}$ coincidence detected in solar flares [4].

As can be seen from Fig. 3, any deviations from the ideal conditions, i.e., those at which enhancement peaks, will result in a reduction in enhancement. This reflects the requirement for a high population of $\mathrm{He}$-like $\mathrm{K}$ and $\mathrm{H}$-like $\mathrm{Cl}$, a necessity for the pumping line to be optically thick, maximizing absorption, and yet low enough density that photopumping is not destroyed by collisional deexcitation [19]. This experiment, relying on long pulse heating of a finite size laser target, will be subject to spatial and temporal gradients in temperature and density. Therefore, the experiment is sampling a range of enhancements from these different points in space and time. A moderate amount of shot-to-shot variation was observed, suggesting that there is a source of noise in the experiment that is poorly understood. In order to establish whether photopumping has occurred, a statistical method of data evaluation was employed, averaging over both the range of conditions that would exist within the plasma, and also that arising from shot-to-shot variations. Generally, limits in repetition rates for high-energy high power laser facilities have precluded the gathering of statistically relevant sample sizes, and such
TABLE I. Summary of shots from the Orion Laser experiment. Note the total incident energy laser is shown, split over multiple beams.

\begin{tabular}{lccc}
\hline \hline $\begin{array}{l}\text { Target } \\
\text { type }\end{array}$ & $\begin{array}{c}\text { Long pulse } \\
\text { energy }(\mathrm{J})\end{array}$ & $\begin{array}{c}\text { Long pulse } \\
\text { duration }(\mathrm{ns})\end{array}$ & $\begin{array}{c}\text { Power } \\
\text { on target }(\mathrm{W})\end{array}$ \\
\hline $\mathrm{KCl}$ & 1043 (4 beams) & 1.5 & $7.0 \times 10^{11}$ \\
$\mathrm{NaCl}$ & 970 (4 beams) & 1.5 & $6.5 \times 10^{11}$ \\
$\mathrm{KCl}$ & 2267 (8 beams) & 1.5 & $1.5 \times 10^{12}$ \\
$\mathrm{NaCl}$ & $2212(8$ beams $)$ & 1.5 & $1.5 \times 10^{12}$ \\
$\mathrm{KF}-\mathrm{KCl}-\mathrm{KF}$ & 1179 (4 beams) & 1.5 & $7.9 \times 10^{11}$ \\
$\mathrm{NaF}-\mathrm{NaCl}-\mathrm{NaF}$ & 1155 (4 beams) & 1.5 & $7.7 \times 10^{11}$ \\
$\mathrm{KF}-\mathrm{KCl}-\mathrm{KF}$ & 1705 (6 beams) & 1.5 & $1.1 \times 10^{12}$ \\
$\mathrm{NaF}-\mathrm{NaCl}-\mathrm{NaF}$ & $1633(6$ beams) & 1.5 & $1.1 \times 10^{12}$ \\
$\mathrm{KF}-\mathrm{KCl}-\mathrm{KF}$ & 1164 (4 beams) & 1 & $1.2 \times 10^{12}$ \\
$\mathrm{NaF}-\mathrm{NaCl}-\mathrm{NaF}$ & 1319 (4 beams) & 1 & $1.3 \times 10^{12}$ \\
$\mathrm{KF}-\mathrm{KCl}-\mathrm{KF}$ & 2121 (8 beams) & 1.5 & $1.4 \times 10^{12}$ \\
$\mathrm{NaF}-\mathrm{NaCl}-\mathrm{NaF}$ & $2302(8$ beams) & 1.5 & $1.5 \times 10^{12}$ \\
$\mathrm{KF}-\mathrm{KCl}-\mathrm{KF}$ & 2347 (8 beams) & 1 & $2.3 \times 10^{12}$ \\
$\mathrm{NaF}-\mathrm{NaCl}-\mathrm{NaF}$ & 2309 (8 beams) & 1 & $2.3 \times 10^{12}$ \\
\hline \hline
\end{tabular}

methods are not typically implemented. However, by focusing on gathering equal pairs of signal and null data, recording identical measurements for each shot, and doing each shot with (relatively) similar experimental setups, it was possible to start studying and quantifying these relatively small signal-tonoise phenomena. The methodology is outlined in the Results and Discussion section.

\section{METHOD}

Up to eight of Orion's $351 \mathrm{~nm}$ wavelength long pulse beams were utilized [25], giving total energies of 1-2.3 kJ on target. The duration of the beams was varied between 1 and $1.5 \mathrm{~ns}$ with short rise times to give a flat top heating profile (see Table I and Fig. 5). Higher energies than predicted were required to achieve the required states. This accounts for losses in heating a real 3D target, compared to the simplified 1D simulation. Phase plates were used on all beams to create a more uniform focal pattern, further assisted by overlapping the beams in time and space. Two laser target configurations were used comprising parylene- $\mathrm{N}$ overcoated microdots (300 $\mu \mathrm{m}$ diameter, close to the focal spot size) with either $\mathrm{KCl}$ or $\mathrm{KF}-\mathrm{KCl}-\mathrm{KF}$ and an $\mathrm{Al}$ flash added for environmental protection of the hydrophilic KF (see Fig. 4). $\mathrm{Na}$ was substituted for $\mathrm{K}$ to produce "null" measurements for comparison, where no such resonances exist between transitions in $\mathrm{Na}$ and $\mathrm{Cl}$. The parylene- $\mathrm{N}$ coating either side of the target provides a tamper layer, which together with the use of a microdot sample aids in reductions of spatial and thermal gradients. This was weighed against a requirement for the microdot diameter to be close to that of the focal spot size, to ensure signal from the $\mathrm{KCl}$ plasma is maximized. The target was heated symmetrically, with half of the long pulse beams incident on each side of the microdot. The main diagnostic, described below, viewed the plasma in the longitudinal direction (see Fig. 5). In doing so it was hoped to 


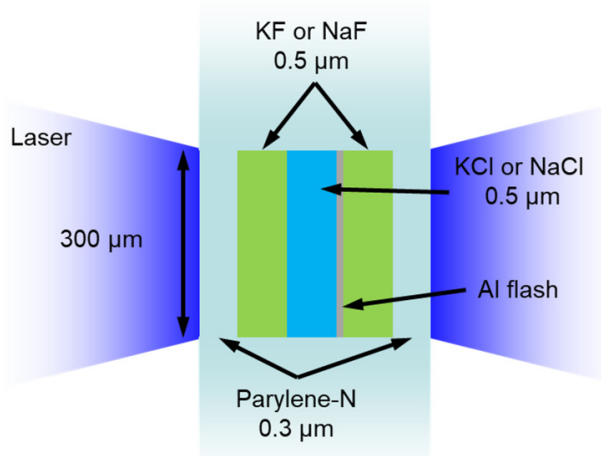

FIG. 4. Slice through the multilayer microdot laser target design. The microdots are encapsulated in an extended sheet of parylene$\mathrm{N}$. A variant, without $\mathrm{KF}$ and with a thicker $(0.8 \mu \mathrm{m}) \mathrm{KCl}$ layer, was also used. The target was heated symmetrically on both sides, as shown in the figure.

predominantly sample the underdense, hot plasma, as required to witness maximum enhancement.

The signature emission was recorded using an extreme ultraviolet grating spectrometer (XUVGS) sensitive in the 120-1200 eV range, coupled to a gated x-ray detector (GXD) for reduced temporal blur [26]. The spectrometer is based on a $2400 \mathrm{lp} / \mathrm{mm}$ variable line spaced Hitachi grating in a Harada design [27], with a nominal spectral resolution of $0.33 \mathrm{eV}$ at $500 \mathrm{eV}$ for the spectrometer and detector combined. The GXD allowed for four temporal strips to be recorded with approximately 200 ps gating pulse (see Fig. 6). As the signal is being recorded on a single instrument, cross calibration is not required; the ratio of signal to null data can be taken as the strength of enhancement. The coincidence was monitored by a suite of x-ray crystal spectrometers, providing time resolved, time integrating, survey, and high spectral resolution K-shell

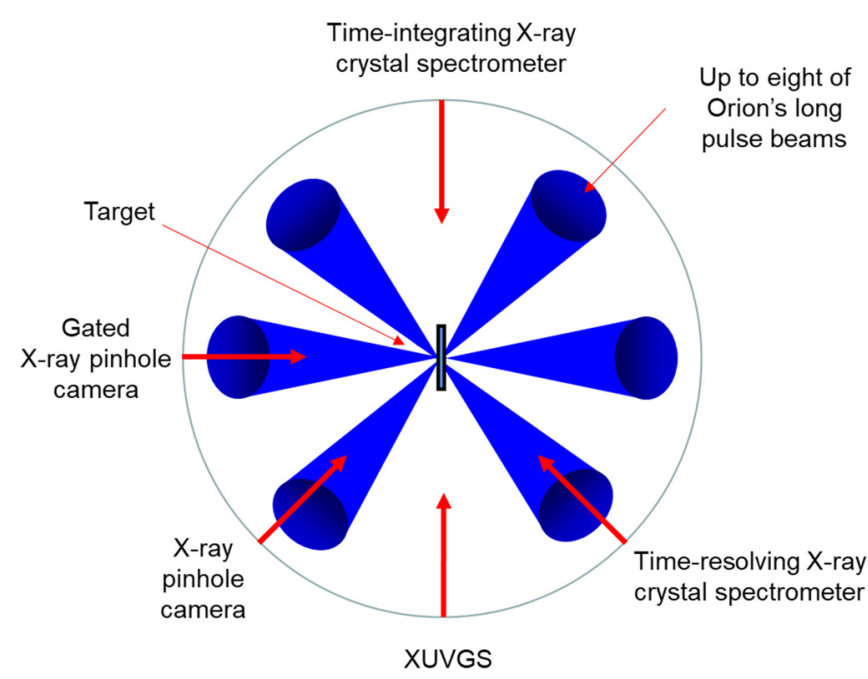

FIG. 5. Setup for the Orion Laser experiment. The Orion long pulses (shown in blue) are incident on either side of the buried layer microdot targets, for symmetrical heating. The signature emission is recorded by the extreme ultraviolet grating spectrometer, approximately transverse to the target normal.

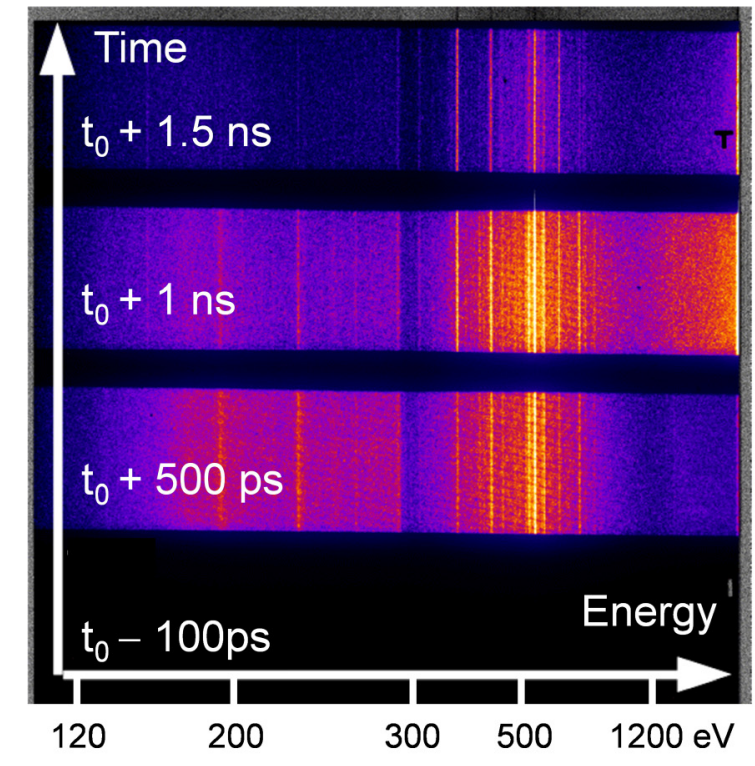

(a)

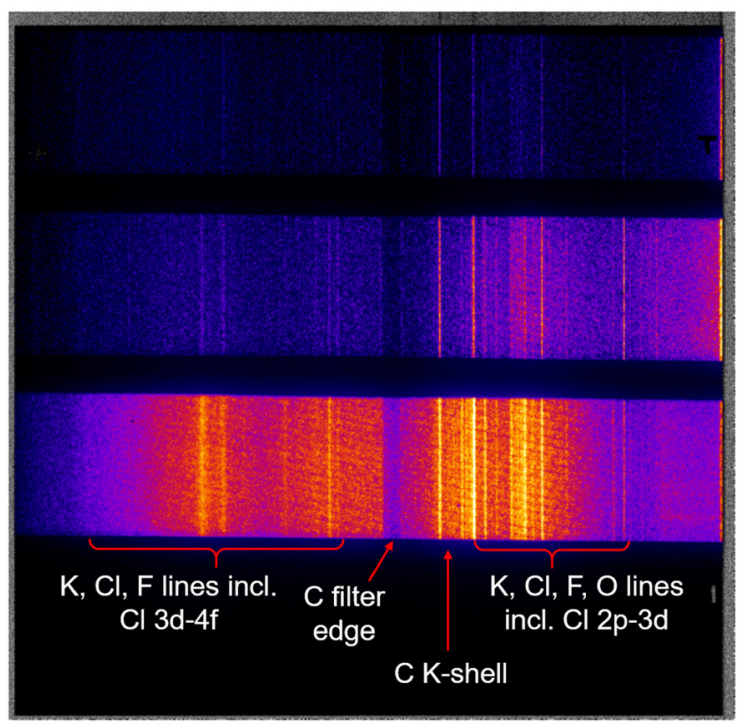

(b)

FIG. 6. XUVGS data recorded onto a GXD for a $\mathrm{KCl}$ (a) and $\mathrm{NaCl}$ (b) plasma, showing the multiple temporal strips. The carbon edge at $284.4 \mathrm{eV}$ is clearly visible and provides a useful energy fiducial. Coupling to the GXD allows multiple frames to be taken of the evolving plasma, significantly reducing temporal blur. By recording data across multiple strips, we can isolate the strip where maximum enhancement is recorded and extract this for further analysis. The interstrip delays were chosen to sample multiple times along the evolution of the plasma, relative to the start of the laser pulse at $t_{0}$. The known dispersion of the XUVGS allows the image pixel value to be converted to energy [see scale on Fig. 6(a)].

data. The Orion High-Resolution X-ray (OHREX) diagnostic [28] clearly resolved the components in the $\mathrm{K} \mathrm{He}_{\alpha}$ line and showed coincidence between the pumped and pumping lines across the full data set. Background subtracted data from a time integrated survey diagnostic gives an average temperature of the emitting plasma of $1-1.5 \mathrm{keV}$. 


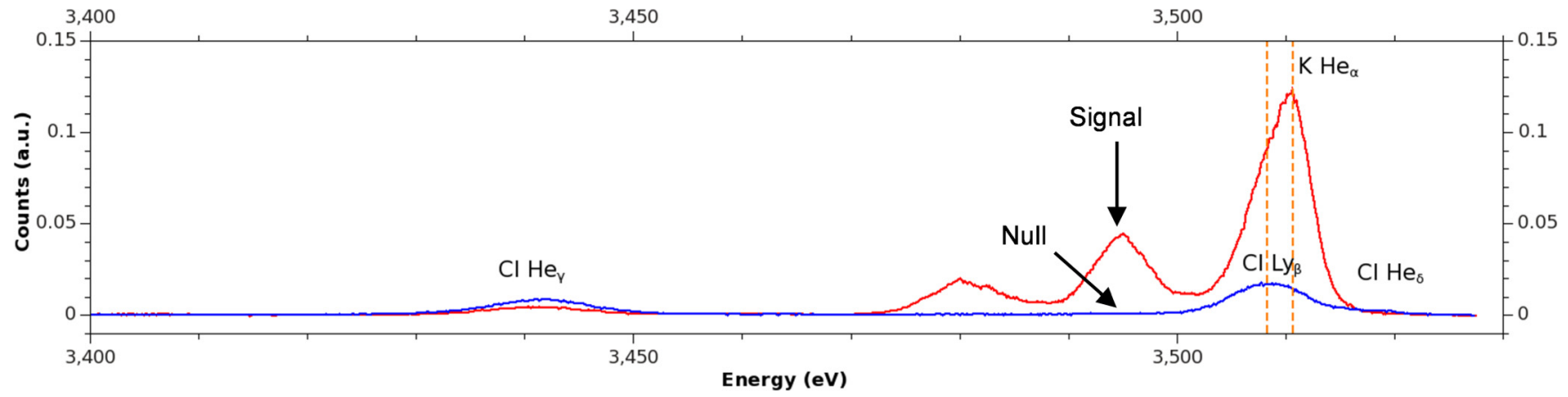

FIG. 7. Orion high-resolution X-ray (OHREX) spectrometer (resolution $E / \Delta E \sim 10000$ ) [28] data showing the $\mathrm{K}_{\mathrm{He}}$ pumping line and $\mathrm{Cl} \mathrm{Ly}{ }_{\beta}$ pump line, the coincidence between which we exploit in this work. The literature values for the line energies are displayed by the orange dashed lines [21]. This data is indicative of all shots taken.

\section{RESULTS AND DISCUSSION}

In total, data from seven shot pairs was collected (see Table I) over a range of energies and pulse durations, all of which are included in the following analysis, encompassing a range of plasma conditions for which we would expect varying levels of LCP. Typical $\mathrm{K}$ and $\mathrm{Cl} \mathrm{K}$-shell data are shown in Fig. 7 and the background corrected data from a single temporal strip is presented in Fig. 8, averaged for each set of seven signal and seven null shots in the region around the signature $\mathrm{Cl} 2 p$ - $3 d$ line. We see a strong contribution from carbon, as well as Li-like potassium, adjacent to the signature line at $546.9 \mathrm{eV}(22.67 \AA)$. In order to enable comparison between signal and null shots, two lines from He-like $\mathrm{Cl}$ which would not undergo photopumping were used to scale between them, identified in the spectra as He-like $\mathrm{Cl} 2 p-3 d$ and $2 s-3 p$ at $479.3 \mathrm{eV}$ and $509.5 \mathrm{eV}$, respectively. Once the scaling factor, taken from an average of the two unpumped lines, was applied, the signature line was isolated and analyzed for enhancement. The result is presented in Fig. 9. We were also able to observe the $2 s-4 p$ line at $741.9 \mathrm{eV}$ $(16.7 \AA)$ and the $3 d-4 f$ line at $191.3 \mathrm{eV}(64.8 \AA)$. The $3 d-4 f$ line was obscured by nearby Li-like potassium emission, and so was unavailable for analysis. The $2 s-4 p$ line, although well resolved, lacked nearby unpumped neighbors to allow for scaling between the signal and null data sets.

First, to increase the signal to noise, we stack the spectra. To provide a confidence level for these measurements, empiri- cal bootstrap analysis was applied to the available data sample [29,30]. The seven sets of normalized data were resampled with replacement from the original seven (e.g., some are repeated), and then the ratio, $x$, of signal to null taken in the vicinity of the signature line recorded each time. This is repeated 10000 times, building up a distribution for what variance in measured average spectrum we would expect if the experiment were repeated multiple times. Figure 9 compares the sample mean, $\bar{x}$, from the original seven samples $x 1, x 2, \ldots, x 7$, with the mean of an empirical bootstrap sample, $\bar{x}^{*}$. The confidence interval is calculated as the basic bootstrap defined as $\left[\bar{x}^{*}-\delta_{\sigma}^{*}, \bar{x}^{*}-\delta_{1-\sigma}^{*}\right]$, where $\delta^{*}=\bar{x}^{*}-x$ and $\delta_{\sigma}^{*}$ denotes the $1 \sigma$ percentiles (16th and 84th) of the bootstrapped ratios $\delta^{*}$ [29]. The calculated enhancement, averaged over the width of the line defined as peak value to $50 \%$ either side, is thereby $[1.39-0.18,1.39+0.16]=[1.21,1.55]$, or an increase of $39 \pm_{18}^{16} \%$. Looking back to Fig. 3 we saw a predicted peak enhancement of $60 \%$. This was, however, calculated for a single condition plasma without inclusion of the temperature and density gradients that would be present in the $\mathrm{KCl}$ plasma. Our conclusions are unlikely to be dramatically different to those obtained using an analytic calculation of the uncertainty, e.g., using a standard error on the mean, or a $t$ distribution, but such methods typically require assuming some knowledge of the noise on the measurements, e.g., that it is Gaussian. Bootstrapping conversely requires no knowledge of the nature of the noise, and also gives a realistic probability

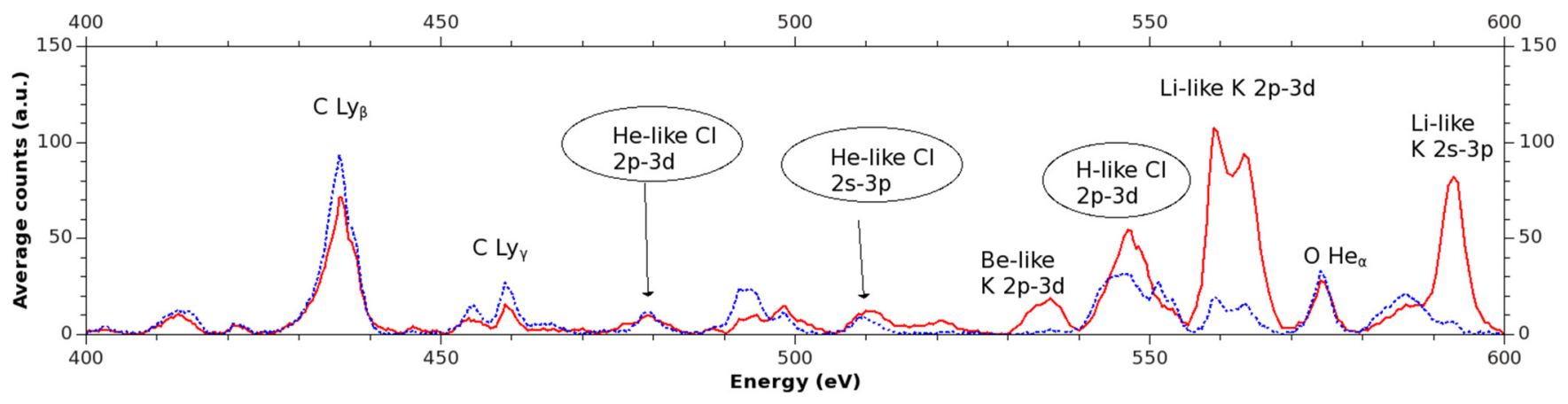

FIG. 8. XUVGS data from a single GXD strip, background corrected and averaged across the seven recorded shots (red solid line). Also shown is the corresponding null data (blue dashed line). Circled are the signature line and the two lines unaffected by photopumping, which were used to scale between the signal and null data sets. 


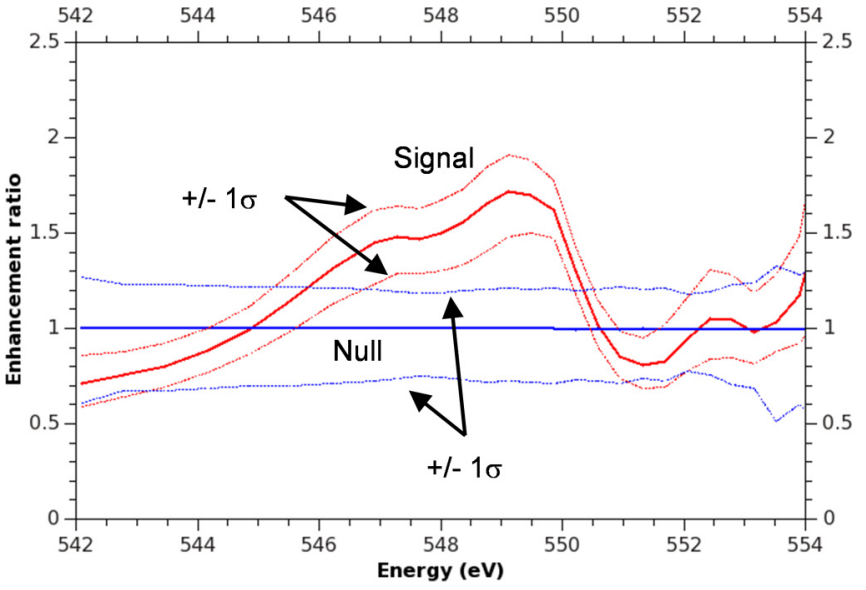

FIG. 9. Enhancement measurements extracted from an average of the seven shot pairs, together with a confidence level provided by the bootstrapping analysis and corresponding analysis for a null scenario. The seven pairs of data were resampled 10000 times to produce the $+/-1 \sigma$ values of enhancement ratio.

distribution from the data with skew, etc., and is simple to implement, and works in a wide variety of circumstances.

The data are presented alongside repeat analysis comparing two sets of null data. As expected, the mean enhancement collapses to 1 , with confidence interval derived from the shot-to-shot variation. The resultant $1 \sigma$ confidence interval is $[0.72,1.21]$, i.e., we would correctly conclude that the enhancement is consistent with 1 when comparing null to null. The probability distribution of the 10000 bootstrapped samples can also be used to estimate the variation of the test statistic if the null hypothesis were true, i.e., that these measurements are indicative of null enhancement (see Fig. 10), and thus calculate a $p$ value. A $p$ value is the probability of getting data as extreme as was measured, or more, if the null hypothesis was true. The basic bootstrap calculates this

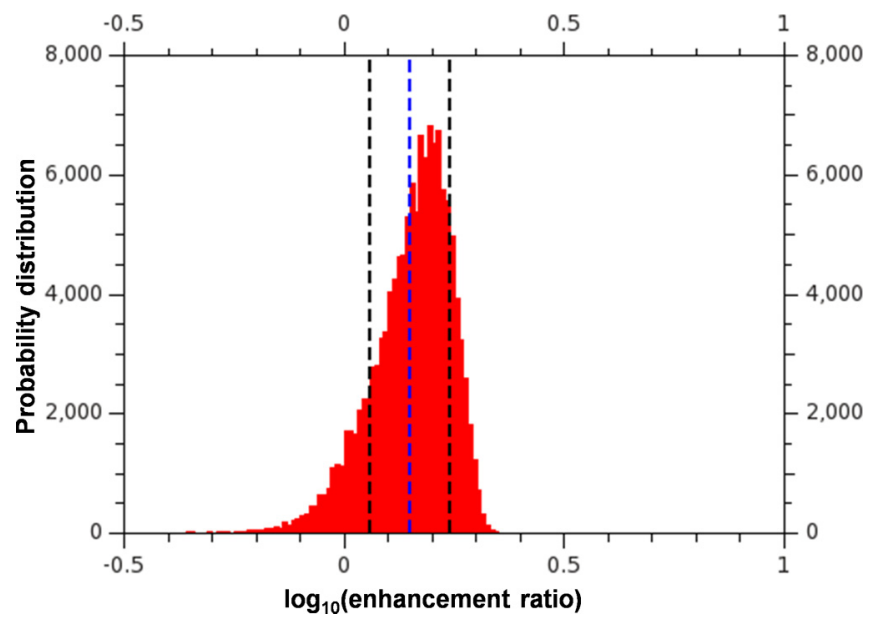

FIG. 10. Probability distribution of enhancement from the bootstrapping analysis. The blue (central) dashed line gives the mean and the black (outer) dashed lines give the $+/-1 \sigma$ bounds taken from the bootstrap analysis and averaged over the full width at half maximum of the line. by finding the probability of recording the sample mean (or greater) in a scenario where true mean of $\log _{10}$ (enhancement ratio) $=0$ is equivalent to finding $P\left(\left|x^{*}\right|>2 x\right)$, the amount greater than two times the sample mean in the distribution given in Fig. 10. The resultant $p$ value is 0.055 . This is relatively modest statistical significance; if there were no enhancement at all, we would still expect to see results we obtained about one time in 20 . This significance certainty would not be sufficient for claiming the existence of nonstandard physics, or for making post-hoc hypotheses (e.g., identifying this enhancement after the data had been collected and inferring photopumping from it). However, given that it is highly implausible that the null hypothesis is true (e.g., we know almost for certain that photopumping is "true"; it is more a question of how large the effect is), and that the size of the observed enhancement is consistent with predictions, it seems reasonable to conclude that we have observed a real enhancement. Finally, we note that the above analysis only accounts for statistical uncertainties, not systematic ones; for example, our estimate of the amount of enhancement occurring would be biased if $\mathrm{NaCl}$ were not a true null and the reference lines behaved differently in the $\mathrm{NaCl}$ and $\mathrm{KCl}$ plasmas.

In terms of astrophysical relevance, observations of enhancement in the $\mathrm{K}-\mathrm{Cl}$ scheme are potentially important for understanding the $\mathrm{x}$-ray spectrum of the intracluster medium, extremely hot gas found in galaxy clusters. Galaxy clusters are the largest virialized objects in the Universe, containing $10^{14}-10^{15} M_{\odot}$ of dark matter, and 10-100 galaxies, and being able to correctly model them is key to our understanding of cosmology and galaxy physics. This cluster hot gas exists as a mixed plasma with $\mathrm{K}$ and $\mathrm{Cl}$ present, of comparable temperatures and relative abundances to that in the experiments, so it may be possible to detect photopumping in the cluster gas too. Intriguingly we note that the $\mathrm{K}$ line pumping the $\mathrm{Cl}$ in the experiment is the same $\mathrm{K}$ line proposed as a possible explanation for the anomalous $3.5 \mathrm{keV}$ observed in galaxy clusters (see Fig. 7 for time-integrated spectra from a $\mathrm{KCl}$ and $\mathrm{NaCl}$ target). The line was initially interpreted as a signal of dark matter; Jeltema et al. [31] point out that by including the $\mathrm{K}$ and $\mathrm{Cl}$ lines there may almost be enough emission to explain these observations, although others have questioned whether the abundances are enough for all the anomalous line intensity to be explained in this way. In addition, more recently a series of experiments (also on Orion) have shown that including a full treatment of satellite lines can lead to corrections of order tens of percent of the strengths of some of the lines in this part of the X-ray spectrum [32]. Although the results presented here do not directly predict an enhancement that could entirely explain the line, it is possible that some combination of geometrical effects and different plasma conditions in the astrophysical case could lead to some previously unanticipated enhancement or modification to specifically the $3.5 \mathrm{keV} \mathrm{K}$ and $\mathrm{Cl}$ lines through a better description of the coupling between the $\mathrm{K}$ and $\mathrm{Cl}$ through the radiation field because of line coincidence.

\section{CONCLUSION}

Analysis of spectral data recorded on the Orion Laser has shown enhancement of emission in $\mathrm{H}$-like $\mathrm{Cl}$, attributable 
to resonant photopumping from a separate ion species. By stacking the data, and using bootstrapping to get realistic uncertainties, we have been able to combine data taken over a number of shots and provide a value of enhancement together with a confidence interval for this measurement.

A mixed plasma with $\mathrm{KCl}$ of comparable temperatures and relative abundances exists astrophysically in the intracluster medium, where understanding the x-ray spectrum of the hot gas is key to identifying any possible dark matter decay signals. Our experiment shows that it may be necessary to include the coupling between ionization levels and the radiation field when modeling the $3.5 \mathrm{keV}$ line part of hot gas spectra in the future. Future work will focus on theoretical and experimental studies of photopumping with plasma parameters more comparable to the intracluster medium, beyond just the temperature and relative abundances, to determine if it is necessary to include in cluster hot gas modeling.

This work will inform our modeling of high energy density plasmas, and it demonstrates the importance of including effects such as resonant photopumping when performing detailed atomic physics calculations of plasmas. Our analysis has relied upon the sophisticated treatment given by CRETIN, in particular the use of physically realistic line broadening models, intensities, and escape factors, that allow reliable modeling of LCP and the radiation field. In doing this work we have increased our understanding of the atomic physics calculations routinely relied on in comparison to experimental plasma physics data and the ongoing interplay between reliance on spectroscopic calculations for plasma diagnosis and the use of this data in verification of theory. This aspect of the work has continued and an extended treatment of LCP modeling is underway.

\section{ACKNOWLEDGMENT}

All data and analysis is $\odot$ British Crown Owned Copyright 2020/AWE.
[1] J. Nilsen, J. H. Scofield, and E. A. Chandler, Reinvestigating the early resonantly photopumped x-ray laser schemes, Appl. Opt. 31, 4950 (1992).

[2] J. Nilsen, D. Burridge, L. Hobbs, D. Hoarty, P. Beiersdorfer, G. Brown, N. Hell, D. Panchenko, M. Gu, A. Saunders et al., Enhanced fluorescence from $\mathrm{x}$-ray line coincidence pumping of $\mathrm{K}$-pumped $\mathrm{Cl}$ and $\mathrm{Mg}$-pumped Ge plasmas, in X-Ray Lasers and Coherent X-Ray Sources: Development and Applications XIII (International Society for Optics and Photonics, Bellingham, WA, 2019), Vol. 11111, p. 1111105.

[3] I. Bowen, The excitation of the permitted O III nebular lines, Publ. Astron. Soc. Pac. 46, 146 (1934).

[4] F. Keenan, K. Poppenhaeger, M. Mathioudakis, S. Rose, J. Flowerdew, D. Hynes, D. Christian, J. Nilsen, and W. Johnson, $\mathrm{X}$-ray line coincidence photopumping in a solar flare, Mon. Not. R. Astron. Soc. 474, 3782 (2017).

[5] N. Qi and M. Krishnan, Photopumping of a C III Ultraviolet Laser by Mn VI Line Radiation, Phys. Rev. Lett. 59, 2051 (1987).

[6] D. O’Neill, C. Lewis, D. Neely, J. Uhomoibhi, M. Key, A. MacPhee, G. Tallents, S. Ramsden, A. Rogoyski, E. McLean et al., Characterisation of soft $\mathrm{x}$-ray amplification observed in Ne-like germanium, Opt. Commun. 75, 406 (1990).

[7] C. A. Back, C. Chenais-Popovics, and R. W. Lee, Photopumping and fluorescence in a laser-produced plasma. I. Experimental results, Phys. Rev. A 44, 6730 (1991).

[8] C. A. Back, J. I. Castor, R. I. Klein, P. G. Dykema, and R. W. Lee, Photopumping and fluorescence in a laser-produced plasma. II. Simulations, Phys. Rev. A 44, 6743 (1991).

[9] A. Gouveia, I. Al'Miev, J. Hawreliak, D. Chambers, T. Liang, R. Marjoribanks, P. A. Pinto, O. Renner, J. Zhang, and J. Wark, Absorption spectroscopy of Al XIII Ly- $\alpha$ radiation by an Fe XXIV plasma, J. Quant. Spectrosc. Radiat. Transfer 81, 199 (2003).

[10] P. Monier, C. Chenais-Popovics, J. P. Geindre, and J. C. Gauthier, Demonstration of quasiresonant x-ray photoexcitation in a laser-created plasma, Phys. Rev. A 38, 2508 (1988).
[11] J. L. Porter, R. B. Spielman, M. K. Matzen, E. J. McGuire, L. E. Ruggles, M. F. Vargas, J. P. Apruzese, R. W. Clark, and J. Davis, Demonstration of Population Inversion by Resonant Photopumping in a Neon Gas Cell Irradiated by a Sodium Z Pinch, Phys. Rev. Lett. 68, 796 (1992).

[12] A. V. Vinogradov, I. I. Sobel'man, and E. Yukov, Possibility of constructing a far-ultraviolet laser utilizing transitions in multiply charged ions in an inhomogeneous plasma, Sov. J. Quantum Electron. 5, 59 (1975).

[13] B. Norton and N. Peacock, Population inversion in laserproduced plasmas by pumping with opacity-broadened lines, J. Phys. B 8, 989 (1975).

[14] V. Bhagavatula, Soft x-ray population inversion by resonant photoexcitation in multicomponent laser plasmas, J. Appl. Phys. 47, 4535 (1976).

[15] W. E. Alley, G. Chapline, P. Kunasz, and J. C. Weisheit, Calculation of gain at $\mathrm{X}$-ray wavelengths resulting from optical pumping of helium-like ions, J. Quant. Spectrosc. Radiat. Transfer 27, 257 (1982).

[16] R. Elton, X-ray Lasers (Academic Press, San Diego, 2012), pp. 99-198.

[17] S. Rose (personal communication).

[18] S. Rose, The non-LTE excitation/ionization code GALAXY, J. Phys. B: At., Mol., Opt. Phys. 31, 2129 (1998).

[19] I. Al'Miev, S. Rose, and J. Wark, Further simulations of the gain in a $\mathrm{K}$ XIX/Cl XVII resonantly photopumped X-ray laser, J. Quant. Spectrosc. Radiat. Transfer 70, 11 (2001).

[20] M. Beer, P. Patel, S. Rose, and J. Wark, Calculations of the modal photon densities and gain in a $\mathrm{K} / \mathrm{Cl}$ resonantly photopumped x-ray laser, J. Quant. Spectrosc. Radiat. Transfer 65, 71 (2000)

[21] V. K. Shen, D. W. Siderius, W. O. Krekelberg, and H. W. Hatch, NIST Standard Reference Simulation Website, NIST Standard Reference Database No. 173 (National Institute of Standards and Technology, Gaithersburg, MD, 2018), http://doi.org/10. 18434/T4M88Q. 
[22] H. A. Scott, Cretin-a radiative transfer capability for laboratory plasmas, J. Quant. Spectrosc. Radiat. Transfer 71, 689 (2001).

[23] M. F. Gu, The flexible atomic code, AIP Conf. Proc. No. 730 (AIP, Melville, NY, 2004), pp. 127-136.

[24] P. Roberts, S. Rose, P. Thompson, and R. Wright, The stability of multiple-shell ICF targets, J. Phys. D 13, 1957 (1980).

[25] N. Hopps, K. Oades, J. Andrew, C. Brown, G. Cooper, C. Danson, S. Daykin, S. Duffield, R. Edwards, D. Egan et al., Comprehensive description of the Orion laser facility, Plasma Phys. Controlled Fusion 57, 064002 (2015).

[26] D. D. Clark, R. Aragonez, T. Archuleta, V. Fatherley, A. Hsu, J. Jorgenson, D. Mares, J. Oertel, K. Oades, P. Kemshall et al., A new gated X-ray detector for the Orion laser facility, in Target Diagnostics Physics and Engineering for Inertial Confinement Fusion (International Society for Optics and Photonics, Bellingham, WA, 2012), Vol. 8505, p. 85050K.

[27] T. Harada, K. Takahashi, H. Sakuma, and A. Osyczka, Optimum design of a grazing-incidence flat-field spectrograph with a spherical varied-line-space grating, Appl. Opt. 38, 2743 (1999).
[28] P. Beiersdorfer, E. Magee, G. Brown, H. Chen, J. Emig, N. Hell, M. Bitter, K. Hill, P. Allan, C. Brown et al., Lineshape spectroscopy with a very high resolution, very high signalto-noise crystal spectrometer, Rev. Sci. Instrum. 87, 063501 (2016).

[29] J. Orloff and J. Bloom, 18.05 Introduction to Probability and Statistics, Massachusetts Institute of Technology: MIT OpenCourseWare, https://ocw.mit.edu. License: Creative Commons BY-NC-SA.

[30] E. Lusso, G. Worseck, J. Hennawi, J. Prochaska, C. Vignali, J. Stern, and J. O'Meara, The first ultraviolet quasar-stacked spectrum at $z \simeq 2.4$ from WFC3, Mon. Not. R. Astron. Soc. 449, 4204 (2015).

[31] T. Jeltema and S. Profumo, Discovery of a $3.5 \mathrm{keV}$ line in the galactic center and a critical look at the origin of the line across astronomical targets, Mon. Not. R. Astron. Soc. 450, 2143 (2015).

[32] M. Weller, P. Beiersdorfer, T. Lockard, G. Brown, A. McKelvey, J. Nilsen, R. Shepherd, V. Soukhanovskii, M. Hill, L. Hobbs et al., Observation of He-like satellite lines of the H-like potassium K XIX emission, Astrophys. J. 881, 92 (2019). 